\title{
高比表面积 $\mathrm{Y}_{2} \mathrm{O}_{3}: \mathrm{Er}^{3+}+\mathrm{TiO}_{2}$ 的制备及上转换发光 特性研究
}

叶岩溪 ${ }^{(1)}$, 刘恩周 ${ }^{(2)}$, 胡晓云 ${ }^{\left(\mathbb{1}^{*}\right.}$, 间志云 ${ }^{(1)}$, 姜振益 ${ }^{(3)}$, 㚞君 ${ }^{(2)}$

(1) 西北大学物理学系, 西安 710069;

(2) 西北大学化工学院, 西安 710069;

(3) 西北大学现代物理研究所, 西安 710069

* 联系人, E-mail: hxy3275@nwu.edu.cn

2011-01-27 收稿, 2011-04-08 接受

国家自然科学基金(20876125)、高等学校博士学科点专项科研基金(20096101110013)、陕西省自然科学基金(2010JZ002)和西北大学研究生 创新基金(09YJC27, 09YJC24)资助项目

摘要 采用共沉淀法和 sol-gel 法制备了 $\mathrm{Y}_{2} \mathrm{O}_{3}: \mathrm{Er}^{3+}, \mathrm{Y}_{2} \mathrm{O}_{3}: \mathrm{Er}^{3+} / \mathrm{Yb}^{3+}$ 和 $\mathrm{Y}_{2} \mathrm{O}_{3}: \mathrm{Er}^{3+}+\mathrm{TiO}_{2}$ 三种样品. 通过扫描电子显微镜(SEM)、透射电子显微镜(TEM)、比表面积仪、紫外可见分光光度计及荧 光光谱仪分析和测试了样品的表面形貌、比表面积、孔隙度、紫外可见吸收光谱和室温下的荧 光光谱. SEM 和 TEM 测试表明共沉淀法制备的样品发光离子分散性很好; sol-gel 法制备的 $\mathrm{Y}_{2} \mathrm{O}_{3}: \mathrm{Er}^{3+}+\mathrm{TiO}_{2}$ 表面分布着许多的介孔, 颗粒直径在 $10 \mathrm{~nm}$ 左右. 对 3 种样品的孔径分布和表 面积测试表明, $\mathrm{Y}_{2} \mathrm{O}_{3}$ : $\mathrm{Er}^{3}$ 和 $\mathrm{TiO}_{2}$ 复合后的性质不是两种物质性质的简单叠加, 其比表面积高达 $135.991 \mathrm{~m}^{2} / \mathrm{g}$, 是纯 $\mathrm{Y}_{2} \mathrm{O}_{3}: \mathrm{Er}^{3+}$ 的 4.8 倍, 是纯 Degussa $\mathrm{P} 25 \mathrm{TiO}_{2}$ 的 2.5 倍, 如此高的比表面积有 利于提高 $\mathrm{TiO}_{2}$ 的光催化性能. 样品的荧光光谱显示其在 388,500 和 $570 \mathrm{~nm}$ 的可见光激发下分 别对应在 237,395 和 $467 \mathrm{~nm}$ 处各有一个上转换发光峰.

关键词

$\mathrm{Y}_{2} \mathrm{O}_{3}: \mathrm{Er}^{3+}$

$\mathrm{TiO}_{2}$

比表面积

上转换发光

多孔材料
$\mathrm{Y}_{2} \mathrm{O}_{3}$ 具有良好的化学和光化学稳定性、高的熔 点、易于实现稀土掺杂等优点 ${ }^{[1]}$, 是一种优良的发光 材料基质; $\mathrm{Er}^{3+}$ 作为发光材料的掺杂离子在可见光区 具有丰富的发射能级. $\mathrm{Y}_{2} \mathrm{O}_{3}: \mathrm{Er}^{3+}$ 不仅具有很好的下转 换发光性能, 同时由于 $\mathrm{Y}_{2} \mathrm{O}_{3}$ 具有约为 $430 \sim 500 \mathrm{~cm}^{-1}$ 的较低声子能量 ${ }^{[1]}$, 其也是一种很好的上转换发光材 料. 上转换发光材料能够在 $980 \mathrm{~nm}$ 红外光和可见光 激发下发射更短波长的光, 因此在三维光学记录技 术、环境照明与显示技术、固体激光器、太阳能电池、 生物标记等方面有广泛的应用 ${ }^{[2 \sim 5]}$.

$\mathrm{TiO}_{2}$ 由于其化学稳定性好、抗磨损性强、成本低、 无毒等特点在光催化制氢、 $\mathrm{CO}_{2}$ 的还原、复杂有机污 染物的降解等方面成为最具有应用潜力的光催化
剂 ${ }^{[6,7]}$. 但是由于 $\mathrm{TiO}_{2}$ 的带隙能较高, 只能吸收占太 阳光不到 5\%的紫外光, 导致其在可见光下光催化活 性很低. 本文把 $\mathrm{Y}_{2} \mathrm{O}_{3}: \mathrm{Er}^{3+}$ 和 $\mathrm{TiO}_{2}$ 复合在一起, 发现 制得样品的比表面积比单独的 $\mathrm{Y}_{2} \mathrm{O}_{3}: \mathrm{Er}^{3+}$ 或者 $\mathrm{TiO}_{2}$ 高很多; $\mathrm{Y}_{2} \mathrm{O}_{3}$ 中的 $\mathrm{Y}^{3+}$ 在复合物中作为掺杂离子, 可以使 $\mathrm{TiO}_{2}$ 的禁带宽度变窄而吸收更多的可见光; 作为基质可以使 $\mathrm{Er}^{3+}$ 吸收长波长的光, 发射短波长 的光使更多的可见光被 $\mathrm{TiO}_{2}$ 吸收. 本研究采用共 沉淀法制备了 $\mathrm{Y}_{2} \mathrm{O}_{3}: \mathrm{Er}^{3+}$ 和 $\mathrm{Y}_{2} \mathrm{O}_{3}: \mathrm{Er}^{3+} / \mathrm{Yb}^{3+}$, 用 sol-gel 制备了 $\mathrm{Y}_{2} \mathrm{O}_{3}: \mathrm{Er}^{3+}+\mathrm{TiO}_{2}$, 研究了其上转换发光特 性, 分析了敏化离子 $\mathrm{Yb}^{3+}$ 对复合材料发光特性的 影响, 并对材料复合前和复合后的比表面积和孔 隙度进行了讨论.

英文版见: Ye Y X, Liu E Z, Hu X Y, et al. Preparation and luminescence properties of $\mathrm{Y}_{2} \mathrm{O}_{3}: \mathrm{Er}^{3+} / \mathrm{TiO}_{2}$ with high specific surface area. Chinese Sci Bull, 2011, 56, doi: 10.1007/s11434-011-4642-5 


\section{1 实验}

( i ) $\mathrm{Y}_{2} \mathrm{O}_{3}: \mathrm{Er}^{3+}$ 和 $\mathrm{Y}_{2} \mathrm{O}_{3}: \mathrm{Er}^{3+} / \mathrm{Yb}^{3+}$ 的制备. 把一定 量 $\mathrm{Y}_{2} \mathrm{O}_{3}$ 和 $\mathrm{Er}_{2} \mathrm{O}_{3}$ 溶解在硝酸中, 在磁力搅拌下加热沸 至澄清, 制备 $\mathrm{Y}_{2} \mathrm{O}_{3}: \mathrm{Er}^{3+} / \mathrm{Yb}^{3+}$ 和 $\mathrm{Y}_{2} \mathrm{O}_{3}: \mathrm{Er}^{3+}$ 的不同之处 在于制备 $\mathrm{Y}_{2} \mathrm{O}_{3}: \mathrm{Er}^{3+} / \mathrm{Yb}^{3+}$ 时, 硝酸溶液变澄清后需要 加人一定量的 $\mathrm{Yb}\left(\mathrm{NO}_{3}\right)_{3}$. 搅拌 $1 \mathrm{~h}$ 后滴人氨水使其沉 淀. 然后再搅拌 $5 \mathrm{~h}$ 后陈化, 陈化一段时间进行离心 洗涤, 先用蒸馏水洗 3 遍, 再用酒精洗 1 遍, 使样品 成中性. 最后干燥退火后即制得样品 $\mathrm{Y}_{2} \mathrm{O}_{3}: \mathrm{Er}^{3+}$ 或 $\mathrm{Y}_{2} \mathrm{O}_{3}: \mathrm{Er}^{3+} / \mathrm{Yb}^{3+}$. 退火步骤分为 3 步: 第一步先升温到 $140^{\circ} \mathrm{C}$, 保温 $1 \mathrm{~h}$, 除去样品中的水; 第二步再升温到 $300^{\circ} \mathrm{C}$, 保温 $1 \mathrm{~h}$ 除去共沉淀法得到的副产物 $\mathrm{NH}_{4} \mathrm{NO}_{3}$; 最后升温到 $600^{\circ} \mathrm{C}$ 或者更高, 保温 $2 \mathrm{~h}$ 使样品结晶.

(ii) $\mathrm{Y}_{2} \mathrm{O}_{3}: \mathrm{Er}^{3+}+\mathrm{TiO}_{2}$ 的制备. 利用正丁醇和冰 乙酸酯化反应生成的水对钛酸丁酯进行水解得到 $\mathrm{TiO}_{2}$. 首先量取一定量的钛酸丁酯和正丁醇, 室温下 摚拌 $0.5 \mathrm{~h}$ 后加人冰乙酸, 然后继续摚拌 $6 \mathrm{~h}$. 与此同 时, 制备 $\mathrm{Er}^{3+}, \mathrm{Y}^{3+}$ 和 $\mathrm{Yb}^{3+}$ 的硝酸化合物, 如( i ) 所述. 然后把澄清的硝酸溶液缓慢滴人搅拌下的钛酸丁酯、 正丁醇和冰乙酸混合液中, 再摚拌 $2 \mathrm{~h}$. 最后进行陈 化, 干燥和退火处理即制得 $\mathrm{Y}_{2} \mathrm{O}_{3}: \mathrm{Er}^{3+}+\mathrm{TiO}_{2}$. 其退火 步骤如 ( i ) 所示, 升温到 $300^{\circ} \mathrm{C}$ 时, 不仅能够除去 $\mathrm{NH}_{4} \mathrm{NO}_{3}$, 而且能够除去由钛酸丁酯分解带来的大部 分有机物.

(iii) 样品的光催化测试. 把 $\mathrm{TiO}_{2}$ 和 $\mathrm{Y}_{2} \mathrm{O}_{3}: \mathrm{Er}^{3+}+$
$\mathrm{TiO}_{2}$ 溶解于水中得到浑浊液倒人自制的反应器中, 控制 $\mathrm{CO}_{2}$ 的流量通人浑浊液中使浑浊液中的样品不 会沉淀, $\mathrm{CO}_{2}$ 持续通人. 在距离反应器 $1 \mathrm{~m}$ 处放置氙 灯作为光源. 通人 $\mathrm{CO}_{2}$, 打开氙灯后光催化还原反应 开始, 反应 $8 \mathrm{~h}$ 后有甲醇生成溶于反应溶液中, 收集 溶液, 测定其甲醇含量.

(iv) 样品的表征. 用 Quamta400FEG 热场发射 环境扫描电子显微镜(SEM) 和 FEI 公司生产的 TF20 型透射电子显微镜(STEM)测试样品的形貌; 用美国 Quantachrome Instruments 生产的 Nova 2000e 型比表 面积仪对样品的比表面积和孔隙度进行测试和分析; 用 UV-3600 型紫外可见分光光度计测得样品的吸收 光谱; 用日立生产的 F-7000 苂光光谱仪测量样品的 苂光光谱, 光源用的是 $150 \mathrm{~W}$ 的氙灯; 用日本岛津公 司 GC-14C 型气相色谱仪测试甲醇的含量, 色谱柱为 PEG-20M 填充柱.

\section{2 结果与讨论}

\section{1 样品的形貌表征及能谱分析}

图 1 为样品的 $\operatorname{EDS}$ 图, 其中 (a)为 $\mathrm{Er}^{3+}$ 摩尔含量 为 $7.5 \%$ 的 $\mathrm{Y}_{2} \mathrm{O}_{3}: \mathrm{Er}^{3+}$, (b) 为 $\mathrm{Er}^{3+} / \mathrm{Yb}^{3+}$ 摩尔含量为 $7.5 \% / 15 \%$ 的 $\mathrm{Y}_{2} \mathrm{O}_{3}: \mathrm{Er}^{3+} / \mathrm{Yb}^{3+}$, 从图中可以看出, (a)含 有 $\mathrm{Y}, \mathrm{O}, \mathrm{Er}$ 等元素, (b)含有 $\mathrm{Y}, \mathrm{O}, \mathrm{Er}, \mathrm{Yb}^{3+}$ 等元素. 插 图表格为各种元素在样品中的重量百分比和原子百 分比, 由其重量百分比算得样品 (a) 的化学式为

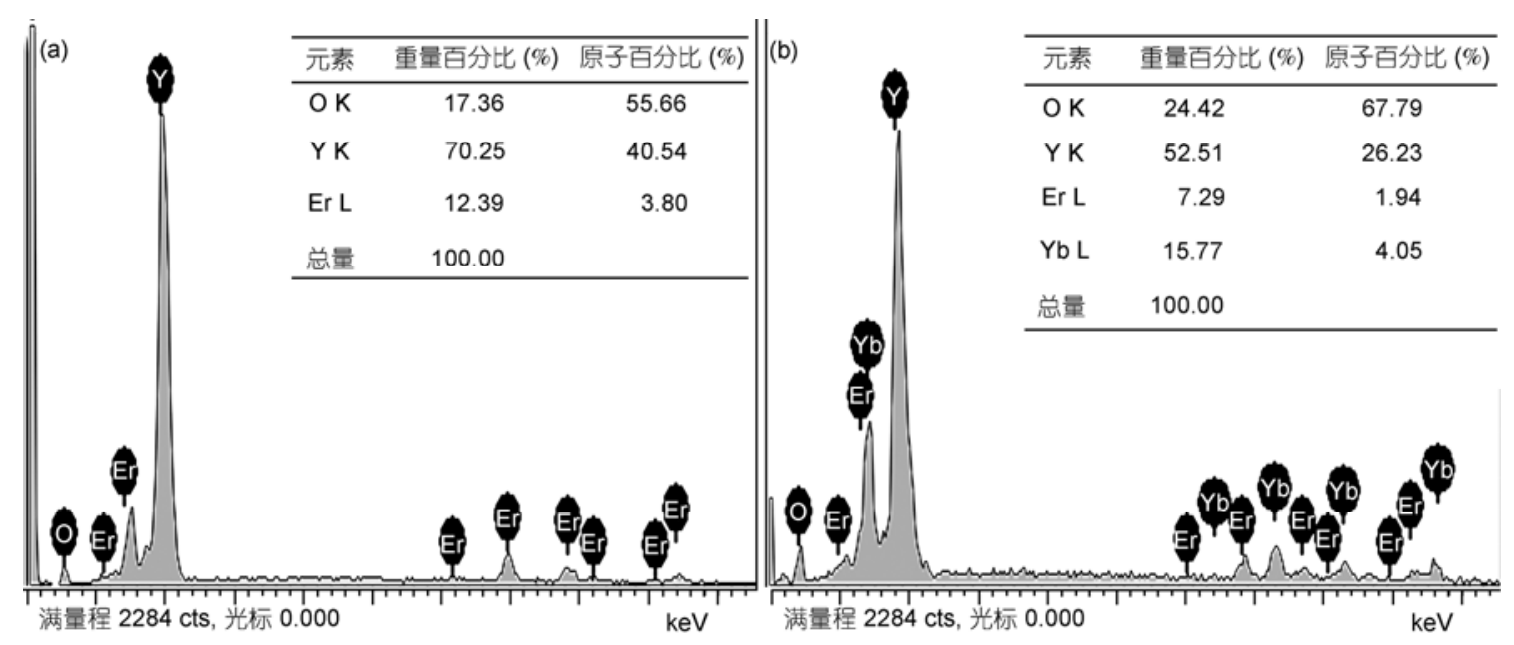

图 1 样品的 EDS 图谱

(a) $\mathrm{Y}_{2} \mathrm{O}_{3}: \mathrm{Er}^{3+}\left(\mathrm{Er}^{3+}\right.$ 摩尔含量为 7.5\%); (b) $\mathrm{Y}_{2} \mathrm{O}_{3}: \mathrm{Er}^{3+} / \mathrm{Yb}^{3+}\left(\mathrm{Er}^{3+} / \mathrm{Yb}^{3+}\right.$ 的摩尔含量为 $\left.7.5 \% / 15 \%\right)$ 
$\mathrm{Y}_{0.79} \mathrm{Er}_{0.07} \mathrm{O}_{1.09}, \mathrm{Er}$ 的摩尔含量为 $8.1 \%$, 样品(b)的化 学式为 $\mathrm{Y}_{0.59} \mathrm{Er}_{0.05} \mathrm{Yb}_{0.09} \mathrm{O}_{1.5}, \mathrm{Er}^{3+}$ 的摩尔含量为 $6.8 \%$, $\mathrm{Yb}^{3+}$ 的摩尔含量为 $12.3 \%$, 这与实验的原始取值符合 得很好. EDS 的计算值和实验取值的相符, 说明制得
样品发光离子具有很好的分散性，这能够避免掺杂 的发光离子和敏化离子聚集在一起造成的苂光猝灭. 从 SEM 图看出, 粉体颗粒团聚在一起形成了大小不 一的较规则小球, 小球外形蓬松(图 2(a)). 为了研究
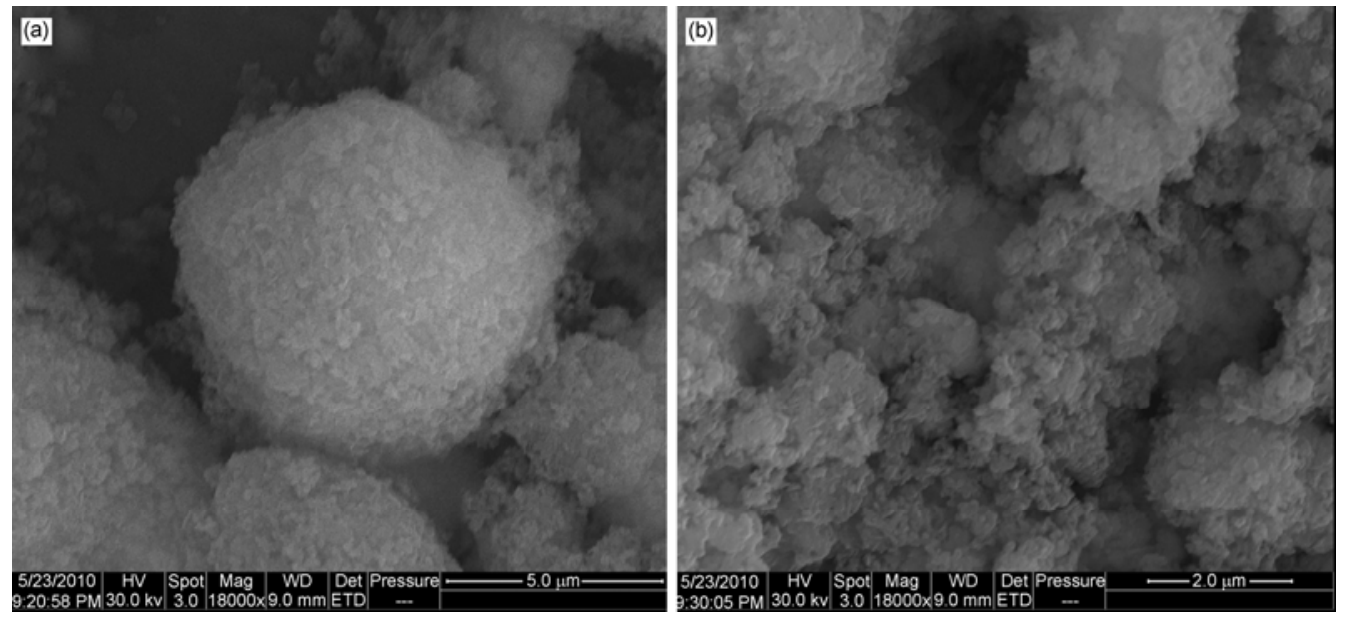

图 2 样品的 SEM 图

(a) $\mathrm{Y}_{2} \mathrm{O}_{3}: \mathrm{Er}^{3+}\left(\mathrm{Er}^{3+}\right.$ 摩尔含量为 7.5\%); (b) $\mathrm{Y}_{2} \mathrm{O}_{3}: \mathrm{Er}^{3+} / \mathrm{Yb}^{3+}\left(\mathrm{Er}^{3+} / \mathrm{Yb}^{3+}\right.$ 的摩尔含量为 7.5\%/15\%)
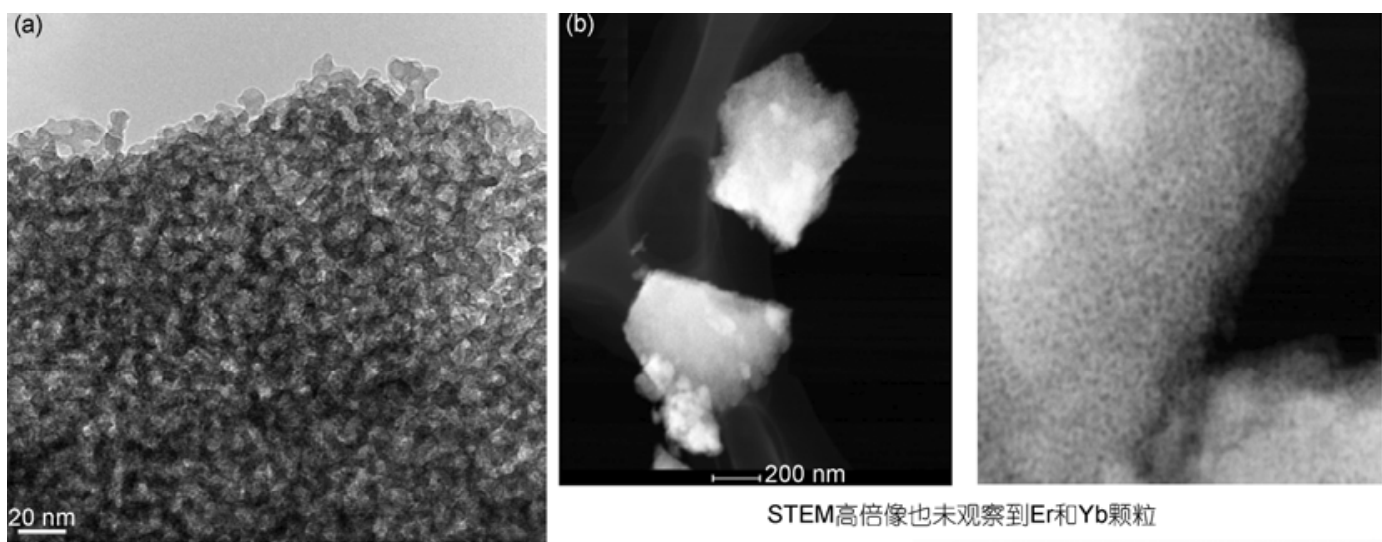

STEM高倍像也未观察到Er和Yb颗粒
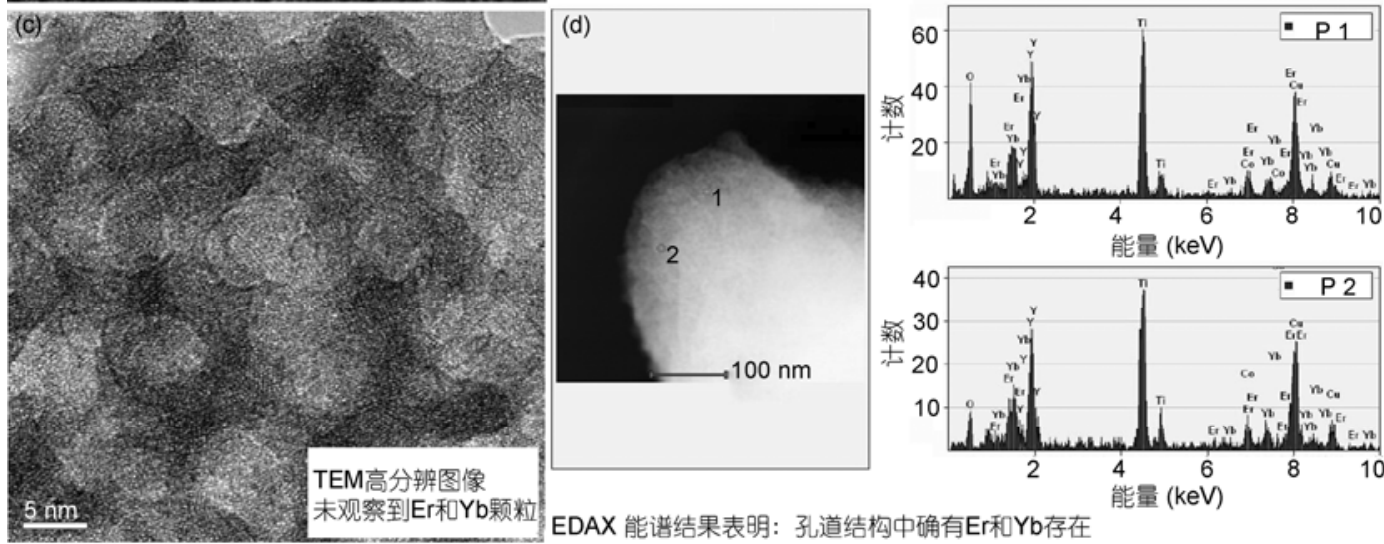

2

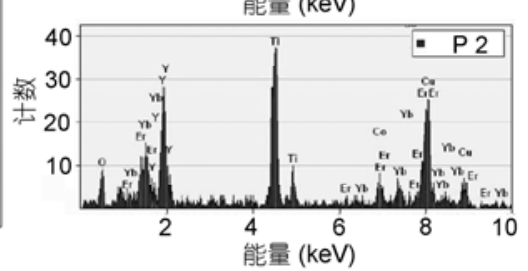

图 $3 \mathrm{Y}_{2} \mathrm{O}_{3}: \mathrm{Er}^{3+} / \mathrm{Yb}^{3+}$ 复合 $\mathrm{TiO}_{2}$ 的 $\mathrm{TEM}$ 图谱

(a) TEM 高倍形貌像; (b) TEM 高分辨图像; (c) STEM 高倍像; (d) EDAX 能谱 
发光离子和敏化离子在复合基质中的位置, 我们对 $\mathrm{Y}_{2} \mathrm{O}_{3}: \mathrm{Er}^{3+} / \mathrm{Yb}^{3+}$ 复合 $\mathrm{TiO}_{2}$ 样品也进行了 TEM 和 STEM 分析, 结果如图 3 所示. 样品颗粒的直径大约为 10 $\mathrm{nm}$, 由于进行退火处理, 样品颗粒团聚在一起以至 于我们不能从 SEM 看出颗粒的大小. 从扫描透射图 片(STEM)(图 3(b)) 可以看出, 样品颗粒表面分布着许 许多多的小孔, 因此我们可以认为复合的样品是一 种多孔材料. 样品的高分辨图像和 STEM 高倍像都 没有观测到 $\mathrm{Er}$ 和 $\mathrm{Yb}$ 颗粒的存在, 而 EDAX 却测试 到了 $\mathrm{Er}^{3+}$ 和 $\mathrm{Yb}^{3+}$ 的存在. 高分辨图像、STEM 高倍像 和 EDAX 说明, 掺杂离子不是分布在颗粒的表面, 而 是进人了颗粒的孔道中和晶格中, 这可以很好地避 免由于表面效应而引起的苂光猝灭, 提高发光效率.

\section{2 样品的比表面积和孔隙度}

图 4 为样品表面的孔径分布图, 带圆圈的曲线表 示样品的累计孔容, 带正方形的曲线表示不同直径 的孔隙的数量分布. 采用 Kelvin 方程, 假设孔径为圆 柱形中孔, 其孔径计算为

$$
r_{k}=\frac{-2 \gamma V_{m}}{R T \ln \left(P / P_{0}\right)},
$$

其中 $\gamma$ 为 $\mathrm{N}_{2}$ 在沸点时的表面张力(在 $77 \mathrm{~K}$ 时, 8.85
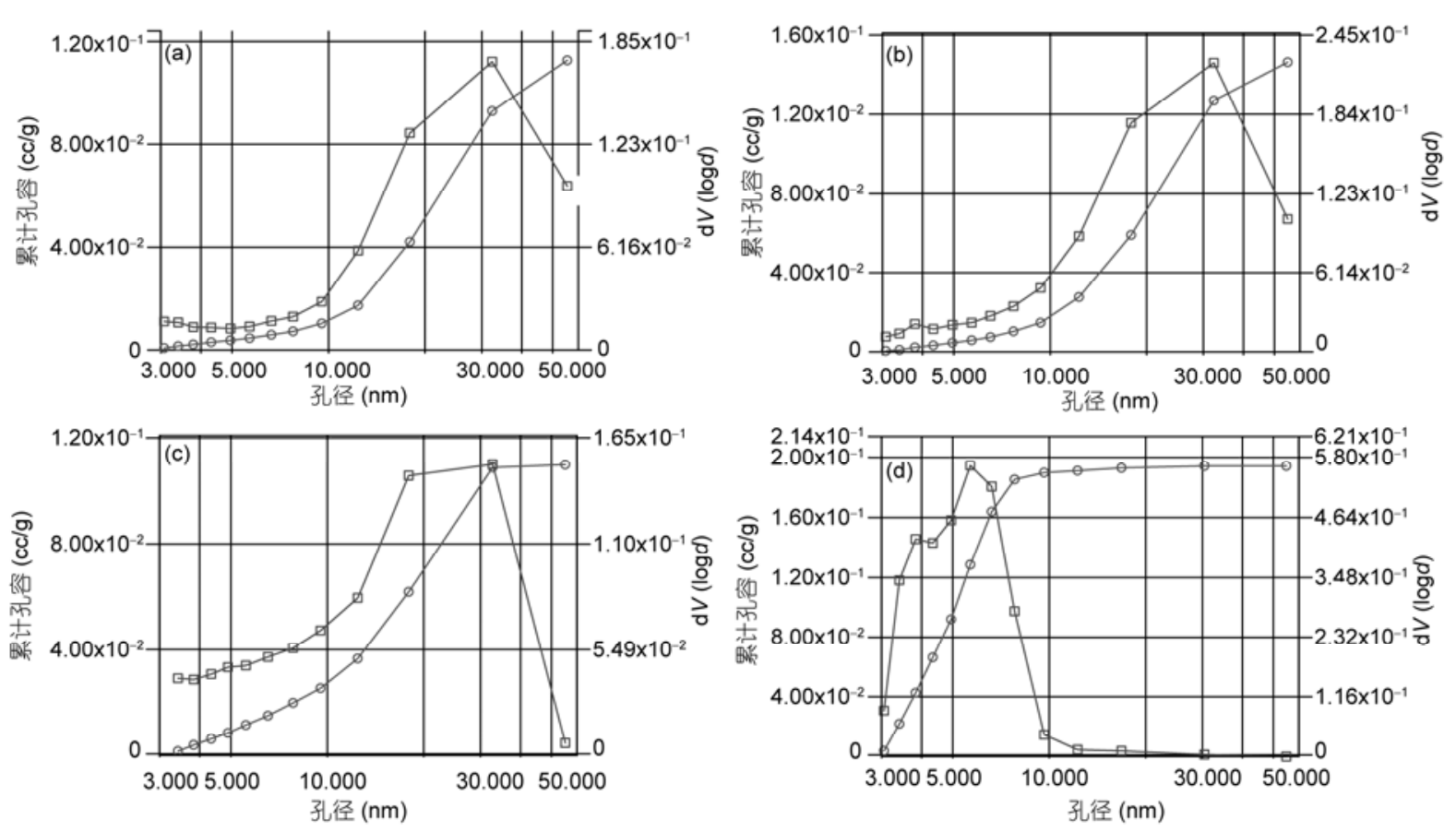

图 4 样品的孔隙分布

(a) $\mathrm{Y}_{2} \mathrm{O}_{3}: \mathrm{Er}^{3+}$; (b) $\mathrm{Y}_{2} \mathrm{O}_{3}: \mathrm{Er}^{3+} / \mathrm{Yb}^{3+}$; (c) Degussa $\mathrm{P} 25 \mathrm{TiO}_{2}$; (d) $\mathrm{Y}_{2} \mathrm{O}_{3}: \mathrm{Er}^{3+}+\mathrm{TiO}_{2}$ $\left.\mathrm{ergs} / \mathrm{cm}^{2}\right), V_{m}$ 为液 $\mathrm{N}_{2}$ 的摩尔体积 $\left(34.7 \mathrm{~cm}^{3} / \mathrm{mol}\right), R$ 为 气体常数 $(8.314 \mathrm{~kJ} /(\mathrm{kmol} \mathrm{K})), T$ 为 $\mathrm{N}_{2}$ 的沸点 $(77 \mathrm{~K})$, $P / P_{0}$ 为 $\mathrm{N}_{2}$ 的相对压力, $r_{k}$ 为孔的 Kelvin 半径. 由图 4 得到的样品的 $\mathrm{N}_{2}$ 最大吸附量, 孔容和数量最多的孔 隙直径数值整理如表 1 所示. $\mathrm{Y}_{2} \mathrm{O}_{3}: \mathrm{Er}^{3+}$ 复合 $\mathrm{TiO}_{2}$ 的孔 容比 $\mathrm{Y}_{2} \mathrm{O}_{3}: \mathrm{Er}^{3+}$ 和 Degussa P25 $\mathrm{TiO}_{2}$ 大得多, 这和 STEM 测试得到的结果是相符合的，也能够证明复合 样品是一种多孔材料的结论是正确的. 由于 $\mathrm{Y}_{2} \mathrm{O}_{3}: \mathrm{Er}^{3+}$ (图 4(a)) 和 $\mathrm{Y}_{2} \mathrm{O}_{3}: \mathrm{Er}^{3+} / \mathrm{Yb}^{3+}$ (图 4(b)) 在小于 50 $\mathrm{nm}$ 时, $\mathrm{N}_{2}$ 吸附量没有最大值, 所以 $\mathrm{N}_{2}$ 最大吸附量取 值在样品孔径为 $50 \mathrm{~nm}$ 处. 图 4 和表 1 说明样品 $\mathrm{Y}_{2} \mathrm{O}_{3}: \mathrm{Er}^{3+}+\mathrm{TiO}_{2}$ 的 $\mathrm{N}_{2}$ 最大吸附量, 孔容和数量最多 的孔隙直径等不是取 $\mathrm{Y}_{2} \mathrm{O}_{3}: \mathrm{Er}^{3+}$ 和 $\mathrm{TiO}_{2}$ 中的一个或者 两者简单的叠加, 而是出现了变化, 具有自己的性质, 已经不是简单的 $\mathrm{Y}_{2} \mathrm{O}_{3}: \mathrm{Er}^{3+}$ 和 $\mathrm{TiO}_{2}$ 在分子水平上的结 合. 这也能侧面说明表 1 中 $\mathrm{Y}_{2} \mathrm{O}_{3}: \mathrm{Er}^{3+}+\mathrm{TiO}_{2}$ 的比表面 积远远大于 $\mathrm{Y}_{2} \mathrm{O}_{3}: \mathrm{Er}^{3+}$ 或 $\mathrm{TiO}_{2}$ 的原因. 表 1 所示的样 品平均孔径表明 4 种样品均是介孔物质(孔径介于 20 500 $\AA$ 的称为中孔或者介孔).

实验测得的 Degussa $\mathrm{P} 25 \mathrm{TiO}_{2}$ 的比表面积为 $53.641 \mathrm{~m}^{2} / \mathrm{g}$, 如表 1 所示, 与 Degussa $\mathrm{P}^{2} 5 \mathrm{TiO}_{2}$ 的标 准比表面积值(为 $(50 \pm 15) \mathrm{m}^{2} / \mathrm{g}$ )相符. 4 种样品的比表 
表 1 样品的比表面积和孔径分布

\begin{tabular}{|c|c|c|c|c|}
\hline 样品 & $\mathrm{Y}_{2} \mathrm{O}_{3}: \mathrm{Er}^{3+}$ & $\mathrm{Y}_{2} \mathrm{O}_{3}: \mathrm{Er}^{3+} / \mathrm{Yb}^{3+}$ & Degussa P25 $\mathrm{TiO}_{2}$ & $\mathrm{Y}_{2} \mathrm{O}_{3}: \mathrm{Er}^{3+}$ 复合 $\mathrm{TiO}_{2}$ \\
\hline 比表面积( $\left.{ }^{2} / g\right)$ & 28.380 & 33.423 & 53.641 & 135.991 \\
\hline 平均孔径(nm) & 17.909 & 17.885 & 3.420 & 3.832 \\
\hline $\mathrm{N}_{2}$ 最大吸附量 $(\mathrm{mL} / \mathrm{g})$ & $1.40 \times 10^{-1}$ & $1.00 \times 10^{-1}$ & $1.00 \times 10^{-1}$ & $1.90 \times 10^{-1}$ \\
\hline 孔容(mL/g) & 0.113 & 0.146 & 0.110 & 0.194 \\
\hline 数量最多的孔隙直径(nm) & 32 & 32 & 32 & 7 \\
\hline
\end{tabular}

面积值如表 1 所示, $\mathrm{Y}_{2} \mathrm{O}_{3}: \mathrm{Er}^{3+}$ 和 $\mathrm{TiO}_{2}$ 复合后的比表面 积是 $\mathrm{Y}_{2} \mathrm{O}_{3}: \mathrm{Er}^{3+}$ 的 4.8 倍, 是 Degussa $\mathrm{P} 25 \mathrm{TiO}_{2}$ 的 2.5 倍. $\mathrm{Y}_{2} \mathrm{O}_{3}: \mathrm{Er}^{3+}+\mathrm{TiO}_{2}$ 高的比表面积将有助于 $\mathrm{TiO}_{2}$ 的光 催化性能的提高.

\section{3 样品的苂光特性}

紫外可见分光光度仪通过对照射在样品上的反 射光的测定得到其吸收光谱. 图 5 为制备的 $\mathrm{Y}_{2} \mathrm{O}_{3}: \mathrm{Er}^{3+}, \mathrm{Y}_{2} \mathrm{O}_{3}: \mathrm{Er}^{3+}+\mathrm{TiO}_{2}$ 与 Degussa P25 $\mathrm{TiO}_{2}$, 纯 $\mathrm{Y}_{2} \mathrm{O}_{3}$ 的紫外可见吸收光谱的比较. $\mathrm{Y}_{2} \mathrm{O}_{3}: \mathrm{Er}^{3+}$ 有 5 个吸 收光谱峰, 峰位分别在 $365,378,489,521,652 \mathrm{~nm}$ 处, 其中 $365 \mathrm{~nm}$ 的强度最弱，几乎不可见. 与纯 $\mathrm{Y}_{2} \mathrm{O}_{3}$ 的 吸收光谱对比可知, $\mathrm{Y}_{2} \mathrm{O}_{3}: \mathrm{Er}^{3+}$ 的 5 个吸收光谱峰都是 属于 $\mathrm{Er}^{3+}$ 的特征吸收峰, $365,378,489,521$ 和 $652 \mathrm{~nm}$ 对应的 $\mathrm{Er}$ 离子能级跃迁分别为 ${ }^{4} \mathrm{I}_{15 / 2} \rightarrow{ }^{4} \mathrm{G}_{9 / 2},{ }^{4} \mathrm{I}_{15 / 2} \rightarrow$ ${ }^{4} \mathrm{G}_{11 / 2},{ }^{4} \mathrm{I}_{15 / 2} \rightarrow{ }^{4} \mathrm{~F}_{7 / 2},{ }^{4} \mathrm{I}_{15 / 2} \rightarrow{ }^{2} \mathrm{H}_{11 / 2}$ 和 ${ }^{4} \mathrm{I}_{9 / 2} \rightarrow{ }^{2} \mathrm{~K}_{15 / 2}$ 的跃迁. $\mathrm{Y}_{2} \mathrm{O}_{3}: \mathrm{Er}^{3+}+\mathrm{TiO}_{2}$ 在波长小于 $400 \mathrm{~nm}$ 时, 出现了极强 的吸收光谱峰, 该吸收峰是由于 $\mathrm{TiO}_{2}$ 的宽禁带宽度 而引起的吸收. 与 $\mathrm{Y}_{2} \mathrm{O}_{3}: \mathrm{E}^{3+}$ 相比, $\mathrm{Y}_{2} \mathrm{O}_{3}: \mathrm{Er}^{3+}+\mathrm{TiO}_{2}$ 中 $\mathrm{Er}^{3+}$ 的特征吸收峰除了被 $\mathrm{TiO}_{2}$ 的宽禁吸收掩盖的 365 和 $378 \mathrm{~nm}$ 吸收峰不能显示出来外, 其他 3 个吸收峰均 出现了. $\mathrm{Y}_{2} \mathrm{O}_{3}: \mathrm{Er}^{3+}+\mathrm{TiO}_{2}$ 中 $\mathrm{Er}^{3+}$ 的特征吸收峰强度

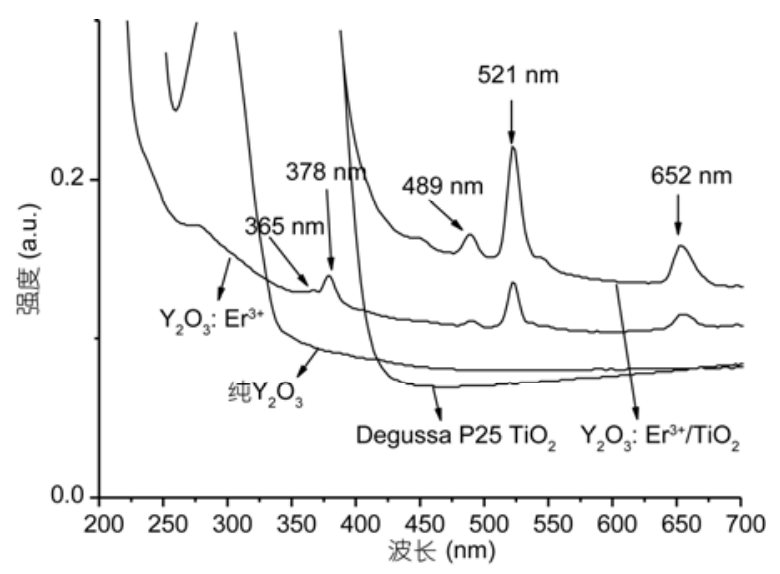

图 5 样品的紫外可见吸收光谱图
明显高于 $\mathrm{Y}_{2} \mathrm{O}_{3}: \mathrm{Er}^{3+}$ 的, 这与复合物高的比表面积有 关，且有利于其光催化性能的提高.

用 5 个吸收峰的峰值作为激发波长对样品进行 激发, 发现在 365,377 和 $521 \mathrm{~nm}$ 激发下出现了下转 换发光现象，在 $388 \mathrm{~nm}$ (处在 $377 \mathrm{~nm}$ 吸收峰曲线的波 腰位置)和 $500 \mathrm{~nm}$ (处在 $521 \mathrm{~nm}$ 吸收峰曲线的波腰位 置)激发下出现了上转换发光现象, 而在 489 和 652 $\mathrm{nm}$ 激发下样品没有任何发光现象. 我们认为, 样品 吸收的 489 和 $652 \mathrm{~nm}$ 光子能量最后转换成了样品的 晶格振动能量和热辐射.

掺杂浓度为 $2 \%$ 、退火温度为 $600{ }^{\circ} \mathrm{C}$ 的 $\mathrm{Y}_{2} \mathrm{O}_{3}: \mathrm{Er}^{3+}$ 在 $388 \mathrm{~nm}$ 激发下出现了 $237 \mathrm{~nm}$ 的紫外光, 在 $500 \mathrm{~nm}$ 激发下出现了 $395 \mathrm{~nm}$ 的紫光, 在 $570 \mathrm{~nm}$ 激发下出现 了 $467 \mathrm{~nm}$ 的蓝光, 如图 6 所示. 上转换发光的反斯托 克定律性质决定了其非常低的发光效率，相应的 3 个 上转换激发波长在紫外可见吸收光谱中都看不出明 显的吸收峰. 由 $\mathrm{Er}^{3+}$ 相应的电子能级值计算得到, 237 , 395 和 $467 \mathrm{~nm}$ 分别为由 ${ }^{4} \mathrm{D}_{3 / 2} \rightarrow{ }^{4} \mathrm{I}_{15 / 2},\left({ }^{2} \mathrm{G},{ }^{4} \mathrm{~F},{ }^{2} \mathrm{H}\right)_{9 / 2} \rightarrow$ ${ }^{4} \mathrm{I}_{15 / 2}$ 和 ${ }^{4} \mathrm{~F}_{5 / 2} \rightarrow{ }^{4} \mathrm{I}_{15 / 2}$ 的跃迁, 其能级图如图 7 所示. 图 8 为 $\mathrm{Y}_{2} \mathrm{O}_{3}: \mathrm{Er}^{3+}, \mathrm{Y}_{2} \mathrm{O}_{3}: \mathrm{Er}^{3+} / \mathrm{Yb}^{3+}$ 和 $\mathrm{Y}_{2} \mathrm{O}_{3}: \mathrm{Er}^{3+}+\mathrm{TiO}_{2}$ 在 3 个激发波长下的上转换发光光谱对比. $\mathrm{Y}_{2} \mathrm{O}_{3}: \mathrm{Er}^{3+}$ 和

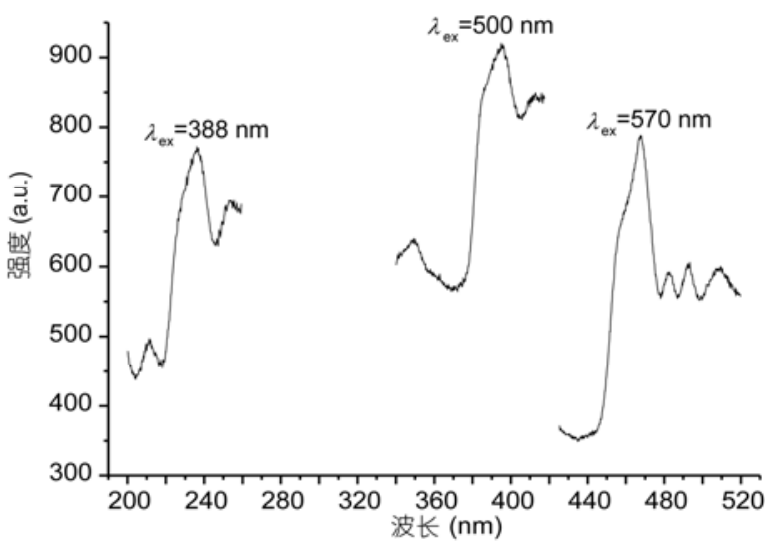

图 $6 \mathrm{Er}^{3+}$ 掺杂浓度为 $2 \%$ 的 $\mathrm{Y}_{2} \mathrm{O}_{3}: \mathrm{Er}^{3+}$ 在不同激发波长的上 转换发光光谱 


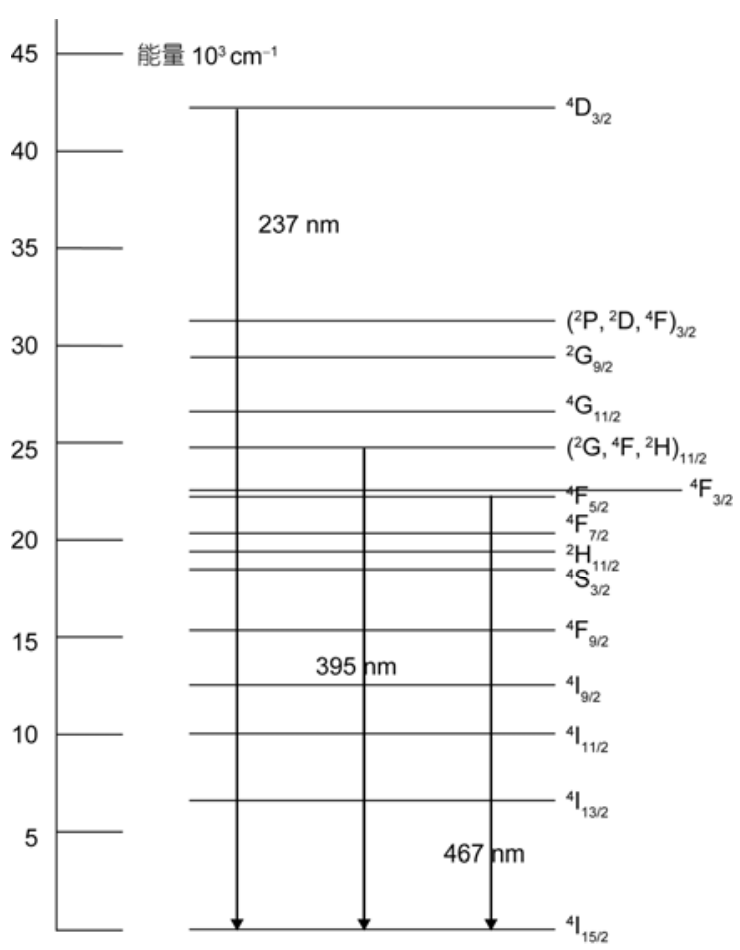

图 $7 \mathbf{E r}^{3+}$ 的能级简图

$\mathrm{Y}_{2} \mathrm{O}_{3}: \mathrm{Er}^{3+} / \mathrm{Yb}^{3+}$ 的对比再次说明在紫外光和可见光激 发下, $\mathrm{Yb}^{3+}$ 不能像在红外光激发下 ${ }^{[2,8,9]}$ 那样对样品的 发光起到很好的敏化作用. $\mathrm{Y}_{2} \mathrm{O}_{3}: \mathrm{Er}^{3+}+\mathrm{TiO}_{2}$ 在 388 , 500 和 $570 \mathrm{~nm}$ 激发下, 同样存在 237, 395 和 $467 \mathrm{~nm}$ 三个发光峰，只是复合基质使得 $\mathrm{Er}^{3+}$ 的发光有所减弱. $\mathrm{Y}_{2} \mathrm{O}_{3}: \mathrm{Er}^{3+}+\mathrm{TiO}_{2}$ 吸收 3 个低能量的光转换成了高能量 的光, 这将有助于提高 $\mathrm{TiO}_{2}$ 的光催化能力.

\section{4 样品光催化还原 $\mathrm{CO}_{2}$ 制备甲醇}

$\mathrm{Y}_{2} \mathrm{O}_{3}: \mathrm{Er}^{3+}$ 和 $\mathrm{Y}_{2} \mathrm{O}_{3}: \mathrm{Er}^{3+}+\mathrm{TiO}_{2}$ 光催化还原 $\mathrm{CO}_{2}$ 制 备甲醇的化学式如下所示:

$$
\mathrm{CO}_{2}+\mathrm{H}_{2} \mathrm{O} \underset{\mathrm{Y}_{2} \mathrm{O}_{3}: \mathrm{Er}^{3+}+\mathrm{TiO}_{2}}{\longrightarrow} \mathrm{CH}_{3} \mathrm{OH}
$$

在实验过程中有一部分样品和 $\mathrm{CO}_{2}$ 的反应液蒸发 后在反应器上方冷凝, 收集后为冷凝液. 表 2 是相同 $\mathrm{TiO}_{2}$ 的量, $\mathrm{TiO}_{2}$ 和 $\mathrm{Y}_{2} \mathrm{O}_{3}: \mathrm{Er}^{3+}+\mathrm{TiO}_{2}$ 光催化还原 $\mathrm{CO}_{2}$ 收集 到的反应液和冷凝液中甲醇含量的对比. $\mathrm{Y}_{2} \mathrm{O}_{3}: \mathrm{Er}^{3+}$

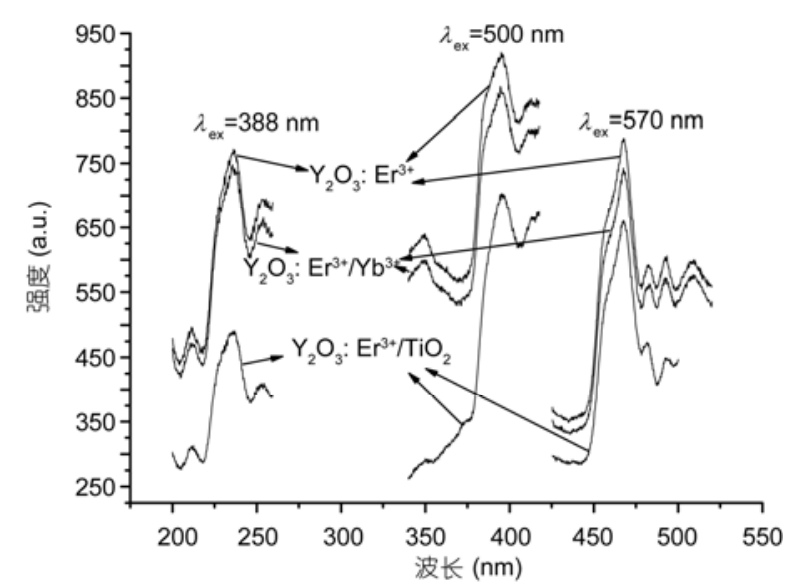

图 $8 \mathrm{Y}_{2} \mathrm{O}_{3}: \mathrm{Er}^{3+}, \mathrm{Y}_{2} \mathrm{O}_{3}: \mathrm{Er}^{3+} / \mathrm{Yb}^{3+}$ 和 $\mathrm{Y}_{2} \mathrm{O}_{3}: \mathrm{Er}^{3+}+\mathrm{TiO}_{2}$ 在不同 激发波长的上转换发光光谱对比

表 $2 \mathrm{TiO}_{2}$ 和 $\mathrm{Y}_{2} \mathrm{O}_{3}: \mathrm{Er}^{3+}+\mathrm{TiO}_{2}$ 光催化还原 $\mathrm{CO}_{2}$ 后的甲醇含量

\begin{tabular}{ccc}
\hline & 纯 $\mathrm{TiO}_{2}$ & $\mathrm{Y}_{2} \mathrm{O}_{3}: \mathrm{Er}^{3+}+\mathrm{TiO}_{2}$ \\
\hline 反应液 $(\mu \mathrm{mol} / \mathrm{g})$ & 47.6 & 79.7 \\
冷凝液 $(\mu \mathrm{mol} / \mathrm{g})$ & 50.0 & 110.7 \\
\hline
\end{tabular}

和 $\mathrm{TiO}_{2}$ 复合后相对于纯 $\mathrm{TiO}_{2}$, 光催化性能有很大的 提高.

\section{3 结论}

用共沉淀法和 sol-gel 法制备了 3 种样品: $\mathrm{Y}_{2} \mathrm{O}_{3}: \mathrm{Er}^{3+}, \mathrm{Y}_{2} \mathrm{O}_{3}: \mathrm{Er}^{3+} / \mathrm{Yb}^{3+}$ 和 $\mathrm{Y}_{2} \mathrm{O}_{3}: \mathrm{Er}^{3+}+\mathrm{TiO}_{2}$. SEM 和 STEM 测试表明共沉淀法制备的样品分散性很好, sol-gel 制备的 $\mathrm{Y}_{2} \mathrm{O}_{3}: \mathrm{Er}^{3+}+\mathrm{TiO}_{2}$ 表面分布着许多的介 孔, 颗粒直径在 $10 \mathrm{~nm}$ 左右. Sol-gel 制备的 $\mathrm{Y}_{2} \mathrm{O}_{3}: \mathrm{Er}^{3+}+\mathrm{TiO}_{2}$ 样品的比表面积高达 $135.991 \mathrm{~m}^{2} / \mathrm{g}$, 远远高于没有复合的 $\mathrm{Y}_{2} \mathrm{O}_{3}: \mathrm{Er}^{3+}$ 或者 $\mathrm{TiO}_{2}$. 对 $\mathrm{Y}_{2} \mathrm{O}_{3}: \mathrm{Er}^{3+}$ 进行紫外可见吸收光谱测试发现, 其在 365, $377,489,521$ 和 $652 \mathrm{~nm}$ 处有吸收峰. 苂光测试发现, 样品在 388,500 和 $570 \mathrm{~nm}$ 的可见光激发分别对应在 237,395 和 $467 \mathrm{~nm}$ 处各有 1 个上转换发光峰. 光催化 还原 $\mathrm{CO}_{2}$ 制备甲醇证明, 加人 $\mathrm{Y}_{2} \mathrm{O}_{3}: \mathrm{Er}^{3+}$ 后, $\mathrm{Y}_{2} \mathrm{O}_{3}: \mathrm{Er}^{3+}+\mathrm{TiO}_{2}$ 的光催化能力比纯的 $\mathrm{TiO}_{2}$ 强.

\section{参考文献}

1 章健, 王世维, 安丽琼, 等. $980 \mathrm{~nm} \mathrm{LD}$ 激发下 $\mathrm{Yb}^{3+}, \mathrm{Er}^{3+}: \mathrm{Y}_{2} \mathrm{O}_{3}$ 纳米晶粉体的上转换发光. 发光学报, 2005, 26: 791-795

2 Yanes A C, Santana-Alonso A, Mendez-Ramos J, et al. $\mathrm{Yb}^{3+}-\mathrm{Er}^{3+}$ co-doped sol-gel transparent nano-glass-ceramics containing NaYF nanocrystals for tuneable up-conversion phosphors. J Alloys Compd, 2009, 480: 706-710 
3 Rapaport A, Milliez J, Bass M, et al. Review of the properties of up-conversion phosphors for new emissive displays. J Display Tech, 2006, 2: 68-78

4 庞涛, 曹望和. 正钽酸钎掺 $\mathrm{Er}^{3+}$ 与 $\mathrm{Er}^{3+}, \mathrm{Yb}^{3+}$ 共掺上转换发光. 科学通报, 2007, 52: 2226-2229

5 Tikhomirov V K, Mortier M. Preparation and up-conversion luminescence of $8 \mathrm{~nm}$ rare-earth doped fluoride nanoparticles. Opt Soc Am, 2008, 16: 14544-14549

6 徐凤秀, 冯光建, 刘素文, 等. 可见光下上转换发光材料掺杂纳米 $\mathrm{TiO}_{2}$ 的光催化活性研究. 硅酸盐通报, 2008, 27: 1140-1145

7 Wang J, Ma T, Zhang G, et al. Preparation of novel nanometer $\mathrm{TiO}_{2}$ catalyst doped withupconversion luminescence agent and investigation on degradation of acid red B dye using visible light. Catal Commun, 2007, 8: 607-611

8 Qiu J B, Song Z G. Nanocrystals precipitation and up-conversion luminescence in $\mathrm{Yb}^{3+}-\mathrm{Tm}^{3+}$ co-doped oxyfluoride glasses. J Rare Earths, 2008, 26: 919-923

9 梁丽芳. 共掺 $\mathrm{Yb}^{3+} / \mathrm{Er}^{3+}\left(\mathrm{or} \mathrm{Tm}^{3+}\right)$ 稀土氟化物粉体的上转换苂光性能以及一些形貌分析. 博士学位论文. 广州: 中山大学, 2005 